Objective: This study intended to compare quality of life (QOL) and psychological aspects among ovarian cancer survivors to healthy female population who presented at Bhumibol Adulyadej Hospital, using standardized questionnaire.

Methods: A cross-sectional analytical study, conducted at Bhumibol Adulyadej Hospital, Thailand from August 2020 to May 2021. This study enrolled healthy Thai female who came to hospital for their health check-up at gynecology clinic as a control group and ovarian cancer survivors on their follow up at tumor clinic as a study group. Questionnaire by Functional Assessment of Cancer Therapy (FACT) was used to measure QOL. Anxiety and depression were assessed by Hospital Anxiety and Depression Scale.

Results: Results shown ovarian cancer survivors and healthy participants had an equal QOL in all four domains of FACT general (FACT-G). Total FACT-G score was similar between two groups (50.2 \pm 9.7 vs. $49.5 \pm 8.5$ ). QOL of nonepithelial ovarian cancer survivors were better than control group $(56.1 \pm 5.4$ vs. $49.5 \pm 8.5$ ) in clinical significance. Psychological aspects were also comparable between two groups.

Conclusion: Ovarian cancer survivors experienced deteriorating in physical function or emotional related to treatment. But after completed the course of treatment, QOL will improve over time along with mental status, approaching level that comparable to the healthy peers.

Poster (M19)

Quality of Life, Nursing \& Supportive Care

https://doi.org/10.3802/jgo.2021.32.S1.M19

\section{Sexual dysfunction in gynecologic cancer survivors}

\section{Apirak Nguanboonmak, "Marut Yanaranop}

Rajavithi Hospital, Bangkok, Thailand (oporakung@hotmail.com)

Objective: To evaluate the sexual dysfunction in gynecologic cancer survivors and to explore the risk factors associated between gynecologic cancer survivors and sexual dysfunction. Methods: A cross-sectional study was conducted with Thai gynecologic cancer survivors in Rajavithi Hospital. To assess the sexual dysfunction, a Thai version of the Female Sexual Function Index (FSFI) questionnaire was utilized. The inclusion criteria were Thai women who were gynecologic cancer survivors scheduled at the Gynecologic Oncology Clinic, Rajavithi Hospital, and had active sexual intercourse (SI). Data were collected between September 1, 2020, to January 31, 2021.

Results: A total of 180 gynecologic cancer survivors who had active SI participated in this study. Those consisted of cervical cancer (43.9\%), endometrial cancer (28.3\%), and ovarian/tubal/ peritoneal cancer $(25.6 \%)$ and gestational trophoblastic neoplasia $(2.2 \%)$. Ninety-nine out of 180 women $(55 \%)$ had sexual dysfunction (FSFI score <26.5). Cervical cancer was found in the majority with sexual dysfunction, but the mean FSFI score was the lowest in endometrial cancer. Among the six domains, desire had the lowest mean score and pain had the highest mean score. Furthermore, postmenopausal status was the significant risk factors that had an impact on sexual dysfunction.

Conclusion: The prevalence of sexual dysfunction in gynecologic cancer survivors was 55\%, and cervical cancer was found in the majority. The factors that significantly affected sexual dysfunction were postmenopausal status.

Poster (M20)

Quality of Life, Nursing \& Supportive Care

https://doi.org/10.3802/jgo.2021.32.S1.M20

\section{Symptom experience of cancer-related cognitive impairment in women with ovarian cancer}

\section{Liying Wang, 'Yi Zhang,' Mei Xue, ' Zhenqi Lu, ${ }^{1}$ Xiaoju Zhang, Yan Ding, ${ }^{2, *}$}

${ }^{1}$ Fudan University Shanghai Cancer Center, Fudan University, Shanghai, China ${ }^{2}$ Department of Nursing, Obstetrics \& Gynecology Hospital of Fudan University, Shanghai, China (ding_yan@fudan.edu.cn)

Objective: To explore Chinese ovarian cancer survivors' symptom experience of cancer-related cognitive impairment (CRCI).

Methods: Semi-structured interviews were conducted with 34 women aged 22-65 in Cancer Center of Fudan University, Shanghai, China. This study was guided by symptom management theory and the conceptual model of preliminary cognition model among cervical cancer survivors. Qualitative content analysis was used to refine themes.

Results: The average age of participants $(\mathrm{n}=34)$ was 51.38 years (range, 25-65 years) and median time since diagnosis was 1 year (range, $0.2-42.8$ years). Three themes and 9 subthemes were identified from the research. For perception of cognitive changes: Become stupid, not as smart as before; not noticing/having the chance to notice any cognition change. Possible influence events in the evaluation of cognitive changes include that comfortable, monotonous, isolation and narrow-focused life cause the brain to "retire"; Surgical anesthesia and chemotherapy were blamed for the change; Fatigue, sleep disorders and anxiety were also considered to affect cognition. For the impact of CRCI, most participants expressed that it was not an issue for them, and they did not particularly care, only a few said that their self-confidence was affected a little. 
Conclusion: The majority of ovarian cancer patients experienced symptoms of CRCI, highlighted by memory and attention problems. Sociocultural and life-and-work adjustments to cope with cancer play an important role in the CRCI symptom experience.

Poster (M21)

Vulvar and Vaginal Cancer

https://doi.org/10.3802/jgo.2021.32.S1.M21

\section{Good outcome vulvar adenocarcinoma- mucinous with pseudomyxoma type stage IVB-grade I after radical vulvectomy, bilateral inguinal lymphadenectomy, left pelvic lymphadenectomy, vulvar reconstruction (keystone flap), colostomy transversum adjuvant radiation}

\section{Sutrisno Sutrisno, Tricia Dewi Anggraeni}

Gynaecology Oncology Division, Dr. Cipto Mangunkusumo Hospital, Jakarta, Indonesia (anggi73@gmail.com)

Objective: To discuss about the role of radical vulvectomy, bilateral inguinal lymphadenectomy, left pelvic lymphadenectomy (external iliac and obturator), vulvar reconstruction (keystone flap), colostomy transversum and adjuvant radiation in vulvar adenocarcinoma of stage IVB - mucinous with pseudomyxoma type: a rare case.

Methods: The 52-year-old woman, para 3, was referred from Adyaksa Hospital with vulvar cancer. Pain in the genital area heavily increased since September 2019. Firstly, at 2017, she complained of mass in her vagina, which was $2 \mathrm{~cm}$ and initially was not painful, but it became bigger in the last 2 months, sized about $8 \mathrm{~cm}$ and felt painful. She also experienced weight loss of 8 $\mathrm{kg}$ in 2 months. She had no complaints on urination or defecation. She consumed pain killer and antibiotics to relieve her pain. In RSUPN Dr. Cipto Mangunkusumo (RSCM), systemic examination was normal, but gynecological examination showed a solid, fixed mass with rough surface covered by skin on the left labia majora $8 \times 8 \times 7 \mathrm{~cm}$, upto $1 / 3$ posterior wall of distal vagina, reddish, with indistinct boundary, easy bleeding, soft, sized $3 \times 4 \mathrm{~cm}$ extended and associated with mass in the vulva. Distance to urethra was $2 \mathrm{~cm}$, distance to anus $1.5 \mathrm{~cm}$. The RSCM histopathology was reported on October 10th, 2019, and it was histologically suitable with well differentiated adenocarcinoma intestinal type of the vulva. Magnetic resonance imaging (MRI) of whole abdomen with contrast RSCM was taken on October 17th, 2019:

- In the vulva region, a solid mass appears to sting contrast with the diffusion restriction measuring $8.7 \times 4.1 \times 7.4 \mathrm{~cm}$. The mass involves the perienum and $1 / 3$ of the distal vagina. OUE, urethra and rectum involvement was not seen. Enlargement of multiple lymph nodes in the bilateral inguinofemoral region (largest diameter on the left side $4.3 \mathrm{~cm}$ ) to bilateral external parailiacs (largest diameter on the left side $1.2 \mathrm{~cm}$ ). Lesions in the inguinofemoral region only involve subcutaneous smears, no visible cuticle defects in the region

- In the left adnexal cystic lesion was seen with a bleeding component of $5.7 \mathrm{~cm}$ in diameter. In the right adnexa there is a cystic lesion of $2 \mathrm{~cm}$ in diameter.

- Uterus anteflexed normal shape and size, does not show mass.

- The liver, gallbladder, spleen, pancreas, kidneys were normal no visible mass or focal lesions.

- Aorta was normal, there is no apparent lymphadenopathy in the paraaorta.On October 24th, 2019, The radical vulvectomy, bilateral inguinal lymphadenectomy, left pelvic lymphadenectomy (external iliac and obturator), vulvar reconstruction (keystone flap), colostomy transversum was done. On February 2020, the adjuvant radiation (28s courses of external beam radiotherapy) was done. At early 2021, colostomy was closed and repair.

Results: On August 5th, 2020, whole abdomen MRI with contrast RSCM was done, revealing no evidence of disease (complete response). At 3-6 months routine evaluation, until September 2021, there was no evidence of disease (complete response). Conclusion: The radical vulvectomy, bilateral inguinal lymphadenectomy, left pelvic lymphadenectomy (external iliac and obturator), vulvar reconstruction (keystone flap), colostomy transversum and adjuvant radiation was the best treatment option for vulvar adenocarcinoma stage IVB - mucinous with pseudomyxoma.

Poster (M22)

Miscellaneous

https://doi.org/10.3802/jgo.2021.32.S1.M22

\section{DNA content in ovarian scaffold using various tissue decellularization techniques: a model for fertility preservation in cancer patients}

Paweena Thuwanut, ${ }^{1, *}$ Christiani A. Amorim, ${ }^{2}$ Wisan Sereepapong, ${ }^{1}$ Pinyada Panyavaranant, ${ }^{1}$ Porntip Sirayapiwat ${ }^{1}$ 'Department of Obstetrics and Gynecology, Faculty of Medicine, Chulalongkorn University, Bangkok, Thailand (Paweena.T@chula.ac.th) ${ }^{2}$ Department of Gynecology, Université Catholique de Louvain, Brussels, Belgium

Objective: The present study principally aimed to compare efficacy of ionic and non-ionic detergents to eliminate DNA content in ovarian scaffold using tissue decellularization technique.

Methods: Ovarian tissues were retrieved from four premenopausal patients, age 42 to 45 years old, with gynaecologic pathological 